\title{
THE SPECTRAL THEOREM FOR UNITARY OPERATORS BASED ON THE $S$-SPECTRUM
}

\author{
DANIEL ALPAY, FABRIZIO COLOMBO, DAVID P. KIMSEY, AND IRENE SABADINI
}

\begin{abstract}
The quaternionic spectral theorem has already been considered in the literature, see e.g. 222, 31, 32, however, except for the finite dimensional case in which the notion of spectrum is associated to an eigenvalue problem, see [21], it is not specified which notion of spectrum underlies the theorem.

In this paper we prove the quaternionic spectral theorem for unitary operators using the $S$-spectrum. In the case of quaternionic matrices, the $S$-spectrum coincides with the right-spectrum and so our result recovers the well known theorem for matrices. The notion of $S$-spectrum is relatively new, see [17, and has been used for quaternionic linear operators, as well as for $n$-tuples of not necessarily commuting operators, to define and study a noncommutative versions of the Riesz-Dunford functional calculus.

The main tools to prove the spectral theorem for unitary operators are the quaternionic version of Herglotz's theorem, which relies on the new notion of $q$-positive measure, and quaternionic spectral measures, which are related to the quaternionic Riesz projectors defined by means of the $S$-resolvent operator and the $S$-spectrum. The results in this paper restore the analogy with the complex case in which the classical notion of spectrum appears in the Riesz-Dunford functional calculus as well as in the spectral theorem.
\end{abstract}

\section{Contents}

1. Introduction

2. Quaternionic Riesz projectors

3. The spectral theorem for quaternionic unitary operators

4. The $S$-spectrum and the spectral theorem

References

\section{IntroduCtion}

One of the main motivations to study spectral theory of linear operators in the quaternionic setting is due to the fact that Birkhoff and von Neumann, see [12, showed that there are essentially two possible settings in which to write the Schrödinger equation:

1991 Mathematics Subject Classification. MSC: 35P05, 47B32, 47S10.

Key words and phrases. Spectral theorem for unitary operators, quaternionic Riesz projectors, $q$-positive measures, quaternionic Herglotz theorem, $S$-spectrum, slice hyperholomorphic functions.

D. Alpay thanks the Earl Katz family for endowing the chair which supported his research. D. P. Kimsey gratefully acknowledges the support of a Kreitman postdoctoral fellowship. F. Colombo and I. Sabadini acknowledge the Center for Advanced Studies of the Mathematical Department of the Ben-Gurion University of the Negev for the support and the kind hospitality during the period in which part of this paper has been written. 
one may use complex-valued functions or one may use quaternion-valued functions. Since then, many efforts have been made by several authors, see [1, 20, 22, 26], to develop a quaternionic version of quantum mechanics.

Fundamental tools in this framework are the theory of quaternionic groups and semigroups on quaternionic Banach spaces which have been studied only recently in the papers [3, 14, 25] using the notion of $S$-spectrum and of $S$-resolvent operator as well as the spectral theorem, which is the main result of this paper.

To fully understand the aim of this work, we start by recalling some basic facts in complex spectral theory. Let $A$ be a linear operator acting on a complex Banach space $X$, and let $\sigma(A)$ and $\rho(A)$ be the spectrum and the resolvent sets of $A$, respectively. One of the most natural ways to associate to a linear operator $A$ the linear operator $f(A)$ is to use the Cauchy formula for holomorphic functions

$$
f(A)=\frac{1}{2 \pi i} \int_{\partial \Omega}(\lambda I-A)^{-1} f(\lambda) d \lambda,
$$

where $\partial \Omega$ is a smooth closed curve that belongs to the resolvent set of $A$ and $f$ a holomorphic function on an open set $\Omega$ which contains the spectrum of $A$. This holomorphic functional calculus is known as Riesz-Dunford functional calculus, see [18].

To any linear operator $A$, it is possible to associate the notion of spectral measures, which can be written explicitly using the Riesz-projectors, as described below. A subset of $\sigma(A)$ that is open and closed in the relative topology of $\sigma(A)$ is called a spectral set. The spectral sets form a Boolean algebra and with each spectral set $\sigma$ one can associate the projector operator

$$
P(\sigma)=\frac{1}{2 \pi i} \int_{C_{\sigma}}(\lambda I-A)^{-1} d \lambda
$$

where $C_{\sigma}$ is a smooth closed curve belonging to the resolvent set $\rho(A)$, such that $C_{\sigma}$ surrounds $\sigma$ but no other points of the spectrum. A spectral measure in the complex Banach space $X$ is then a homomorphic map of the Boolean algebra of the sets into the Boolean algebra of projection operators in $X$ which has the additional property that it maps the unit in its domain into the identity operator in its range.

As is well known, the spectrum $\sigma(A)$ appearing in the definition of the Riesz-projectors $P(\sigma)$ is the same spectrum on which is supported the spectral measure $E(\lambda)$ appearing in the spectral theorem for normal linear operators in a complex Hilbert space. Precisely, for a normal linear operator $B$ on a Hilbert space, given a continuous function $g$ on the spectrum $\sigma(B)$, we have

$$
g(B)=\int_{\sigma(B)} g(\lambda) d E(\lambda) .
$$

In the quaternionic setting, before the introduction of the $S$-spectrum, see [17, two spectral problems were considered. We discuss the case of a right linear quaternionic operator (the case of a left linear operator being similar) $T: \mathcal{V} \rightarrow \mathcal{V}$ acting on a quaternionic two sided Banach space $\mathcal{V}$, that is $T\left(w_{1} \alpha+w_{2} \beta\right)=T\left(w_{1}\right) \alpha+T\left(w_{2}\right) \beta$, for $\alpha, \beta \in \mathbb{H}$ and $w_{1}, w_{2} \in \mathcal{V}$. The symbol $\mathcal{B}^{R}(\mathcal{V})$ denotes the Banach space of bounded right linear operators.

The left spectrum $\sigma_{L}(T)$ of $T$ is related to the resolvent operator $(s \mathcal{I}-T)^{-1}$ that is

$$
\sigma_{L}(T)=\left\{s \in \mathbb{H} \quad: \quad s \mathcal{I}-T \quad \text { is not invertible in } \mathcal{B}^{R}(\mathcal{V})\right\}
$$


where the notation $s \mathcal{I}$ in $\mathcal{B}^{R}(\mathcal{V})$ means that $(s \mathcal{I})(v)=s v$.

The right spectrum $\sigma_{R}(T)$ of $T$ is associated with the right eigenvalue problem, i.e. the search for nonzero vectors satisfying $T(v)=v s$. It is important to note that if $s$ is an eigenvalue, then all quaternions belonging to the sphere $r^{-1} s r, r \in \mathbb{H} \backslash\{0\}$, are also eigenvalues. But observe that the operator $\mathcal{I} s-T$ associated to the right eigenvalue problem is not linear, so it is not clear what is the resolvent operator to be considered. A natural notion of spectrum that arises in the definition of the quaternionic functional calculus is the one of $S$-spectrum. In the case of matrices, the $S$-spectrum coincides with the set of right eigenvalues; in the general case of a linear operator, the point $S$-spectrum coincides with the set of right eigenvalues.

In the literature there are several papers on the quaternionic spectral theorem, see e.g. [22, 32, however the notion of spectrum in use is not made clear. Recently, there has been a resurgence of interest in this topic, see [24], where the authors prove the spectral theorem, based on the $S$-spectrum, for compact normal operators on a quaternionic Hilbert space. In this paper we prove the quaternionic spectral theorem for unitary operators using the $S$-spectrum, which is then realized to be the correct notion of spectrum for the quaternionic spectral theory of unitary operators.

The $S$-spectrum, see [17], is defined as

$$
\sigma_{S}(T)=\left\{s \in \mathbb{H} \quad: \quad T^{2}-2 \operatorname{Re}(s) T+|s|^{2} \mathcal{I} \quad \text { is not invertible }\right\},
$$

while the $S$-resolvent set is

$$
\rho_{S}(T):=\mathbb{H} \backslash \sigma_{S}(T)
$$

where $s=s_{0}+s_{1} i+s_{2} j+s_{3} k$ is a quaternion, $i, j$ and $k$ are the imaginary units of the quaternion $s, \operatorname{Re}(s)=s_{0}$ is the real part and the norm $|s|$ is such that $|s|^{2}=$ $s_{0}^{2}+s_{1}^{2}+s_{2}^{2}+s_{3}^{2}$. Due to the noncommutativity of the quaternions, there are two resolvent operators associated with a quaternionic linear operator: the left and the right $S$-resolvent operators which are defined as

$$
S_{L}^{-1}(s, T):=-\left(T^{2}-2 \operatorname{Re}(s) T+|s|^{2} \mathcal{I}\right)^{-1}(T-\bar{s} \mathcal{I}), \quad s \in \rho_{S}(T)
$$

and

$$
S_{R}^{-1}(s, T):=-(T-\bar{s} \mathcal{I})\left(T^{2}-2 \operatorname{Re}(s) T+|s|^{2} \mathcal{I}\right)^{-1}, \quad s \in \rho_{S}(T),
$$

respectively. Using the notion of $S$-spectrum and the notion of slice hyperholomorphic functions, see Section 4, we can define the quaternionic functional calculus, see [15, 16, 17. We point out that the $S$-resolvent operators are also used in Schur analysis in the realization of Schur functions in the slice hyperholomorphic setting see [6, 7, 8] and [2, 10] for the classical case.

To set the framework in which we will work, we give some preliminaries. Consider the complex plane $\mathbb{C}_{I}:=\mathbb{R}+I \mathbb{R}$, for $I \in \mathbb{S}$, where $\mathbb{S}$ is the unit sphere of purely imaginary quaternions. Observe that $\mathbb{C}_{I}$ can be identified with a complex plane since $I^{2}=-1$ for every $I \in \mathbb{S}$. Let $\Omega \subset \mathbb{H}$ be a suitable domain that contains the $S$-spectrum of $T$. We define the quaternionic functional calculus for left slice hyperholomorphic functions $f: \Omega \rightarrow \mathbb{H}$ as

$$
f(T)=\frac{1}{2 \pi} \int_{\partial\left(\Omega \cap \mathbb{C}_{I}\right)} S_{L}^{-1}(s, T) d s_{I} f(s),
$$


where $d s_{I}=-d s I$; for right slice hyperholomorphic functions, we define

$$
f(T)=\frac{1}{2 \pi} \int_{\partial\left(\Omega \cap \mathbb{C}_{I}\right)} f(s) d s_{I} S_{R}^{-1}(s, T) .
$$

These definitions are well posed since the integrals depend neither on the open set $\Omega$ nor on the complex plane $\mathbb{C}_{I}$. Using a similar idea, we define the projection operators which will provide the link between the spectral theorem and the $S$-spectrum.

Our proofs will make use of a quaternionic version of Herglotz's theorem proved in the recent paper [5]. This theorem will be the starting point to prove the quaternionic spectral theorem for unitary operators, in analogy with the classical case.

We have proved that if $U$ is a unitary operator acting on a quaternionic Hilbert space $\mathcal{H}$, then, for $x, y \in \mathcal{H}$, there exists a spectral measure $E$ defined on the Borel sets of $[0,2 \pi)$ such that for every slice continuous function $f \in \mathcal{S}\left(\sigma_{S}(U)\right)$, we have

$$
\langle f(U) x, y\rangle=\int_{0}^{2 \pi} f\left(e^{I t}\right)\langle d E(t) x, y\rangle, \quad x, y, \in \mathcal{H} .
$$

Moreover, for $t$ belonging to the Borel sets of $[0,2 \pi)$, the measures

$$
\nu_{x, y}(t)=\langle E(t) x, y\rangle, \quad x, y \in \mathcal{H},
$$

are related to the $S$-spectrum of $U$ by the quaternionic Riesz projectors through the relation

$$
\mathcal{P}\left(\sigma_{S}^{0}(U)\right)=E\left(t_{1}\right)-E\left(t_{0}\right),
$$

where $\sigma_{S}^{0}(U)$ is the spectral set in the unit circle in $\mathbb{C}_{I}$ delimited by the angles $t_{0}, t_{1}$. The plan of the paper is the following. In Section 2, we introduce the quaternionic Riesz projectors. Section 3 contains the proof of the main result of the paper, namely the spectral theorem for the unitary operatrs. In Section 4, we discuss the relation with the $S$-spectrum.

\section{Quaternionic Riesz Projectors}

In the following we denote by $\mathcal{B}(\mathcal{V})$ the space of bounded quaternionic linear operators on the left or on the right, since the results of this section hold in both cases.

The classical Riesz projectors are a powerful tool in spectral analysis and the study of such projectors is based on the resolvent equation. Recently, in the paper [4], it has been shown that there exists a $S$-resolvent equation but in the quaternionic setting it involves both the $S$-resolvent operators. Precisely we have:

Theorem 2.1 (The $S$-resolvent equation). Let $T \in \mathcal{B}(\mathcal{V})$ and let $s$ and $p \in \rho_{S}(T)$. Then we have

$S_{R}^{-1}(s, T) S_{L}^{-1}(p, T)=\left(\left(S_{R}^{-1}(s, T)-S_{L}^{-1}(p, T)\right) p-\bar{s}\left(S_{R}^{-1}(s, T)-S_{L}^{-1}(p, T)\right)\right)\left(p^{2}-2 s_{0} p+|s|^{2}\right)^{-1}$,

but also

$S_{R}^{-1}(s, T) S_{L}^{-1}(p, T)=\left(s^{2}-2 p_{0} s+|p|^{2}\right)^{-1}\left(s\left(S_{R}^{-1}(s, T)-S_{L}^{-1}(p, T)\right)-\left(S_{R}^{-1}(s, T)-S_{L}^{-1}(p, T)\right) \bar{p}\right)$.

The quaternionic functional calculus is defined on the class of slice hyperholomorphic functions $f: \Omega \subseteq \mathbb{H} \rightarrow \mathbb{H}$. Such functions have a Cauchy formula, that works on specific domains which are called axially symmetric slice domain. On this Cauchy formula is based the quaternionic functional calculus. 
If we consider an element $I$ in the unit sphere of purely imaginary quaternions

$$
\mathbb{S}=\left\{q=i x_{1}+j x_{2}+k x_{3} \text { such that } x_{1}^{2}+x_{2}^{2}+x_{3}^{2}=1\right\}
$$

then $I^{2}=-1$, and for this reason the elements of $\mathbb{S}$ are also called imaginary units. Note that $\mathbb{S}$ is a 2-dimensional sphere in $\mathbb{R}^{4}$. Given a nonreal quaternion $p=x_{0}+\operatorname{Im}(p)=$ $x_{0}+I|\operatorname{Im}(p)|, I=\operatorname{Im}(p) /|\operatorname{Im}(p)| \in \mathbb{S}$, we can associate to it the 2-dimensional sphere defined by

$$
[p]=\left\{x_{0}+I|\operatorname{Im}(p)|: I \in \mathbb{S}\right\} .
$$

For any fixed $I \in \mathbb{S}$, the set $\mathbb{C}_{I}=\{u+I v: u, v \in \mathbb{R}\}$ can be identified with the complex plane $\mathbb{C}$.

Definition 2.2 (Axially symmetric slice domain). Let $\Omega$ be a domain in $\mathbb{H}$. We say that $\Omega$ is a slice domain (s-domain for short) if $\Omega \cap \mathbb{R}$ is non empty and if $\Omega \cap \mathbb{C}_{I}$ is a domain in $\mathbb{C}_{I}$ for all $I \in \mathbb{S}$. We say that $\Omega$ is axially symmetric if, for all $q \in \Omega$, the 2 -sphere $[q]$ is contained in $\Omega$.

Definition 2.3. An axially symmetric set $\sigma \subseteq \sigma_{S}(T)$ which is both open and closed in $\sigma_{S}(T)$ in its relative topology, is called a $\mathrm{S}$-spectral set. For sake of simplicity we will call it a spectral set.

The definition of a S-spectral set is suggested by the symmetry properties of the $S$ spectrum. In fact, if $p \in \sigma_{S}(T)$, then all of the elements of the 2-sphere $[p]$ are contained in $\sigma_{S}(T)$.

Definition 2.4. Let $T$ be a quaternionic linear operator on a quaternionic Banach space $\mathcal{V}$. Denote by $\Omega_{\sigma}$ an axially symmetric s-domain that contains the spectral set $\sigma$ but not any other points of the $S$-spectrum. Suppose that the Jordan curves $\partial\left(\Omega_{\sigma} \cap \mathbb{C}_{I}\right)$ belong to the $S$-resolvent set $\rho_{S}(T)$, for every $I \in \mathbb{S}$. We define the family $\mathcal{P}(\sigma)$ of quaternionic operators, depending on the spectral sets $\sigma$, as

$$
\mathcal{P}(\sigma)=\frac{1}{2 \pi} \int_{\partial\left(\Omega_{\sigma} \cap \mathbb{C}_{I}\right)} S_{L}^{-1}(s, T) d s_{I} .
$$

The operators $\mathcal{P}(\sigma)$ are called (quaternionic) Riesz projectors.

Remark 2.5. The definition of $\mathcal{P}(\sigma)$ can be given using the right $S$-resolvent operator $S_{R}^{-1}(s, T)$, that is

$$
\mathcal{P}(\sigma)=\frac{1}{2 \pi} \int_{\partial\left(\Omega_{\sigma} \cap \mathbb{C}_{I}\right)} d s_{I} S_{R}^{-1}(s, T) .
$$

Using the left $S$-resolvent operator we define the Riesz projectors associated with the $S$-spectrum. In [4, Theorem 3.19] we proved that $\mathcal{P}(\sigma)$ is a projector and that it commutes with $T$.

The following lemma will be useful in the sequel.

Lemma 2.6. Let $B \in \mathcal{B}(\mathcal{V})$ and let $\Omega$ be an axially symmetric s-domain. If $p \in \Omega$, then

$$
\frac{1}{2 \pi} \int_{\partial\left(\Omega \cap \mathbb{C}_{I}\right)} d s_{I}(\bar{s} B-B p)\left(p^{2}-2 s_{0} p+|s|^{2}\right)^{-1}=B .
$$


Moreover, if $s \in \Omega$, then

$$
\frac{1}{2 \pi} \int_{\partial\left(\Omega \cap \mathbb{C}_{I}\right)}(\bar{s} B-B p)\left(p^{2}-2 s_{0} p+|s|^{2}\right)^{-1} d p_{I}=-B .
$$

Proof. It follows the same lines of the proof of Lemma 3.18 in [4].

Theorem 2.7. Let $T$ be a quaternionic linear operator. Then the family of operators $\mathcal{P}(\sigma)$ has the following properties:

(i) $(\mathcal{P}(\sigma))^{2}=\mathcal{P}(\sigma)$;

(ii) $T \mathcal{P}(\sigma)=\mathcal{P}(\sigma) T$;

(iii) $\mathcal{P}\left(\sigma_{S}(T)\right)=\mathcal{I}$;

(iv) $\mathcal{P}(\emptyset)=0$;

(v) $\mathcal{P}(\sigma \cup \delta)=\mathcal{P}(\sigma)+\mathcal{P}(\delta) ; \quad \sigma \cap \delta=\emptyset$

(vi) $\mathcal{P}(\sigma \cap \delta)=\mathcal{P}(\sigma) \mathcal{P}(\delta)$.

Proof. Properties (i) and (ii) are proved in Theorem 3.19 in [4]. Property (iii) follows from the quaternionic functional calculus since

$$
T^{m}=\frac{1}{2 \pi} \int_{\partial\left(\Omega \cap \mathbb{C}_{I}\right)} S_{L}^{-1}(s, T) d s_{I} s^{m}, \quad m \in \mathbb{N}_{0}
$$

for $\sigma_{S}(T) \subset \Omega$, which for $m=0$ gives

$$
\mathcal{I}=\frac{1}{2 \pi} \int_{\partial\left(\Omega \cap \mathbb{C}_{I}\right)} S_{L}^{-1}(s, T) d s_{I}
$$

Property (iv) is a consequence of the functional calculus as well.

Property (v) follows from

$$
\begin{aligned}
\mathcal{P}(\sigma \cup \delta) & =\frac{1}{2 \pi} \int_{\partial\left(\Omega_{\sigma \cup \delta} \cap \mathbb{C}_{I}\right)} S_{L}^{-1}(s, T) d s_{I} \\
& =\frac{1}{2 \pi} \int_{\partial\left(\Omega_{\sigma} \cap \mathbb{C}_{I}\right)} S_{L}^{-1}(s, T) d s_{I}+\frac{1}{2 \pi} \int_{\partial\left(\Omega_{\delta} \cap \mathbb{C}_{I}\right)} S_{L}^{-1}(s, T) d s_{I} \\
& =\mathcal{P}(\sigma)+\mathcal{P}(\delta) .
\end{aligned}
$$

To prove (vi), assume that $\sigma \cap \delta \neq \emptyset$ and consider

$$
\begin{aligned}
\mathcal{P}(\sigma) \mathcal{P}(\delta) & =\frac{1}{(2 \pi)^{2}} \int_{\partial\left(\Omega_{\sigma} \cap \mathbb{C}_{I}\right)} d s_{I} S_{R}^{-1}(s, T) \int_{\partial\left(\Omega_{\delta} \cap \mathbb{C}_{I}\right)} S_{L}^{-1}(p, T) d p_{I} \\
& =\frac{1}{(2 \pi)^{2}} \int_{\partial\left(\Omega_{\sigma} \cap \mathbb{C}_{I}\right)} d s_{I} \int_{\partial\left(\Omega_{\delta} \cap \mathbb{C}_{I}\right)}\left[S_{R}^{-1}(s, T)-S_{L}^{-1}(p, T)\right] p\left(p^{2}-2 s_{0} p+|s|^{2}\right)^{-1} d p_{I} \\
& -\frac{1}{(2 \pi)^{2}} \int_{\partial\left(\Omega_{\sigma} \cap \mathbb{C}_{I}\right)} d s_{I} \int_{\partial\left(\Omega_{\delta} \cap \mathbb{C}_{I}\right)} \bar{s}\left[S_{R}^{-1}(s, T)-S_{L}^{-1}(p, T)\right]\left(p^{2}-2 s_{0} p+|s|^{2}\right)^{-1} d p_{I},
\end{aligned}
$$

where we have used the $S$-resolvent equation (see Theorem 2.1). We rewrite the above relation as

$$
\begin{aligned}
\mathcal{P}(\sigma) \mathcal{P}(\delta) & =-\frac{1}{(2 \pi)^{2}} \int_{\partial\left(\Omega_{\sigma} \cap \mathbb{C}_{I}\right)} d s_{I} \int_{\partial\left(\Omega_{\delta} \cap \mathbb{C}_{I}\right)}\left[\bar{s} S_{R}^{-1}(s, T)-S_{R}^{-1}(s, T) p\right]\left(p^{2}-2 s_{0} p+|s|^{2}\right)^{-1} d p_{I} \\
& +\frac{1}{(2 \pi)^{2}} \int_{\partial\left(\Omega_{\sigma} \cap \mathbb{C}_{I}\right)} d s_{I} \int_{\partial\left(\Omega_{\delta} \cap \mathbb{C}_{I}\right)}\left[\bar{s} S_{L}^{-1}(p, T)-S_{L}^{-1}(p, T) p\right]\left(p^{2}-2 s_{0} p+|s|^{2}\right)^{-1} d p_{I} \\
& :=\mathcal{J}_{1}+\mathcal{J}_{2} .
\end{aligned}
$$


Now thanks to Lemma 2.6 and Remark 2.5 we have

$$
\begin{aligned}
\mathcal{J}_{1} & =-\frac{1}{(2 \pi)^{2}} \int_{\partial\left(\Omega_{\sigma} \cap \mathbb{C}_{I}\right)} d s_{I} \int_{\partial\left(\Omega_{\delta} \cap \mathbb{C}_{I}\right)}\left[\bar{s} S_{R}^{-1}(s, T)-S_{R}^{-1}(s, T) p\right]\left(p^{2}-2 s_{0} p+|s|^{2}\right)^{-1} d p_{I} \\
& =\frac{1}{2 \pi} \int_{\partial\left(\Omega_{\sigma} \cap \mathbb{C}_{I}\right)} d s_{I} S_{R}^{-1}(s, T), \text { for } \quad s \in \Omega_{\delta} \cap \mathbb{C}_{I} \\
& =\frac{1}{2 \pi} \int_{\partial\left(\Omega_{\sigma} \cap \mathbb{C}_{I}\right)} S_{L}^{-1}(s, T) d s_{I}, \quad \text { for } \quad s \in \Omega_{\delta} \cap \mathbb{C}_{I}
\end{aligned}
$$

while $\mathcal{J}_{1}=0$ when $s \notin \Omega_{\delta} \cap \mathbb{C}_{I}$ since

$$
\int_{\partial\left(\Omega_{\delta} \cap \mathbb{C}_{I}\right)}\left[\bar{s} S_{R}^{-1}(s, T)-S_{R}^{-1}(s, T) p\right]\left(p^{2}-2 s_{0} p+|s|^{2}\right)^{-1} d p_{I}=0 .
$$

Similarly, one can show that

$$
\mathcal{J}_{2}=\frac{1}{2 \pi} \int_{\partial\left(\Omega_{\delta} \cap \mathbb{C}_{I}\right)} S_{L}^{-1}(p, T) d p_{I}, \quad \text { for } \quad p \in \Omega_{\sigma} \cap \mathbb{C}_{I}
$$

while $\mathcal{J}_{2}=0$ when $p \notin \Omega_{\sigma} \cap \mathbb{C}_{I}$. The integrals $\mathcal{J}_{1}, \mathcal{J}_{2}$ are either both zero or both nonzero, so with a change of variable we get

$$
\mathcal{J}_{1}+\mathcal{J}_{2}=\frac{1}{2 \pi} \int_{\partial\left(\Omega_{\sigma \cap \delta} \cap \mathbb{C}_{I}\right)} S_{L}^{-1}(r, T) d r_{I}=\mathcal{P}(\sigma \cap \delta)
$$

From now on we will always work in quaternionic Hilbert spaces, so we will recall some definitions.

Let $\mathcal{H}$ be a right linear quaternionic Hilbert space with an $\mathbb{H}$-valued inner product $\langle\cdot, \cdot\rangle$ which satisfies

$$
\begin{gathered}
\langle x, y\rangle=\overline{\langle y, x\rangle} ; \\
\langle x, x\rangle \geq 0 \quad \text { and }\|x\|^{2}:=\langle x, x\rangle=0 \Longleftrightarrow x=0 ; \\
\langle x \alpha+y \beta, z\rangle=\langle x, z\rangle \alpha+\langle y, z\rangle \beta ; \\
\langle x, y \alpha+z \beta\rangle=\bar{\alpha}\langle x, z\rangle+\bar{\beta}\langle x, z\rangle
\end{gathered}
$$

for all $x, y, z \in \mathcal{H}$ and $\alpha, \beta \in \mathbb{H}$. Any right linear quaternionic Hilbert space can be made also a left linear space, by fixing an Hilbert basis, see [23], Section 3.1. We call an operator $A$ from the right quaternionic Hilbert space $\mathcal{H}_{1}$, with inner product $\langle\cdot, \cdot\rangle_{1}$, to another right quaternionic Hilbert space $\mathcal{H}_{2}$, with inner product $\langle\cdot, \cdot\rangle_{2}$, right linear if

$$
A(x \alpha+y \beta)=(A x) \alpha+(A y) \beta,
$$

for all $x, y$ in the domain of $A$ and $\alpha, \beta \in \mathbb{H}$. We call an operator $A$ bounded if

$$
\|A\|:=\sup _{\|x\| \leq 1}\|A x\|<\infty .
$$

Corresponding to any bounded right linear operator $A: \mathcal{H}_{1} \rightarrow \mathcal{H}_{2}$ there exists a unique bounded right linear operator $A^{*}: \mathcal{H}_{2} \rightarrow \mathcal{H}_{1}$ such that

$$
\langle A x, y\rangle_{2}=\left\langle x, A^{*} y\right\rangle_{1}
$$

and $\|A\|=\left\|A^{*}\right\|$ (see Proposition 6.2 in [1] $)$. 
Let $\mathcal{H}$ be a right quaternionic Hilbert space with inner product $\langle\cdot, \cdot\rangle$. We call a right linear operator $U: \mathcal{H} \rightarrow \mathcal{H}$ unitary if

$$
\left\langle U^{*} U x, y\right\rangle=\langle x, y\rangle, \quad \text { for all } x, y \in \mathcal{H},
$$

or, equivalently, $U^{-1}=U^{*}$.

Theorem 2.8. Let $\mathcal{H}$ be a right linear quaternionic Hilbert space and let $U$ be a unitary operator on $\mathcal{H}$. Then the $S$-spectrum of $U$ belongs to the unit sphere of the quaternions.

Proof. See Theorem 4.8 in [23].

By $\mathbf{B}([0,2 \pi))$ we denote the Borel sets in $[0,2 \pi)$.

Lemma 2.9. Let $x, y \in \mathcal{H}$ and let $\mathcal{P}(\sigma)$ be the projector associated with the unitary operator $U$. We define

$$
m_{x, y}(\sigma):=\langle\mathcal{P}(\sigma) x, y\rangle, \quad x, y \in \mathcal{H}, \quad \sigma \in \mathbf{B}([0,2 \pi)) .
$$

Then the $\mathbb{H}$-valued measures $m_{x, y}$ defined on $\mathbf{B}([0,2 \pi))$ enjoy the following properties

(i) $m_{x \alpha+y \beta, z}=m_{x, z} \alpha+m_{y, z} \beta$;

(ii) $m_{x, y \alpha+z \beta}=\bar{\alpha} m_{x, y}+\bar{\beta} m_{x, z}$;

(iii) $m_{x, y}([0,2 \pi)) \leq\|x\|\|y\|$,

where $x, y, z \in \mathcal{H}$ and $\alpha, \beta \in \mathbb{H}$.

Proof. Properties (i) and (ii) follow from the properties of the quaternionic scalar product, while (iii) follows from Property (iii) in Theorem 2.7 and the Cauchy-Schwarz inequality (see Lemma 5.6 in [1]]).

\section{THE SPECTRAL THEOREM FOR QUATERNIONIC UNITARY OPERATORS}

We recall some classical results and also their quaternionic analogs which will be useful to prove a spectral theorem for quaternionic unitary operators.

Theorem 3.1 (Herglotz's theorem). The function $n \mapsto r(n)$ from $\mathbb{Z}$ into $\mathbb{C}^{s \times s}$ is positive definite if and only if there exists a unique $\mathbb{C}^{s \times s}$-valued measure $\mu$ on $[0,2 \pi)$ such that

$$
r(n)=\int_{0}^{2 \pi} e^{i n t} d \mu(t), \quad n \in \mathbb{Z} .
$$

Given $P \in \mathbb{H}^{s \times s}$, there exist unique $P_{1}, P_{2} \in \mathbb{C}^{s \times s}$ such that $P=P_{1}+P_{2} j$. Recall the bijective homomorphism $\chi: \mathbb{H}^{s \times s} \rightarrow \mathbb{C}^{2 s \times 2 s}$ given by

$$
\chi P=\left(\begin{array}{cc}
P_{1} & P_{2} \\
-\bar{P}_{2} & \bar{P}_{1}
\end{array}\right) \quad \text { where } P=P_{1}+P_{2} j
$$

Definition 3.2. Given a $\mathbb{H}^{s \times s}$-valued measure $\nu$, we may always write $\nu=\nu_{1}+\nu_{2} j$, where $\nu_{1}$ and $\nu_{2}$ are uniquely determined $\mathbb{C}^{s \times s}$-valued measures. We call a measure $d \nu$ on $[0,2 \pi)$ q-positive if the $\mathbb{C}^{2 s \times 2 s}$-valued measure

$$
\mu=\left(\begin{array}{ll}
\nu_{1} & \nu_{2} \\
\nu_{2}^{*} & \nu_{3}
\end{array}\right), \quad \text { where } \nu_{3}(t)=\nu_{1}(2 \pi-t), \quad t \in[0,2 \pi)
$$

is positive and, in addition,

$$
\nu_{2}(t)=-\nu_{2}(2 \pi-t)^{T}, \quad t \in[0,2 \pi) .
$$


Remark 3.3. If $\nu$ is $q$-positive, then $\nu=\nu_{1}+\nu_{2} j$, where $\nu_{1}$ is a uniquely determined positive $\mathbb{C}^{s \times s}$-valued measure and $\nu_{2}$ is a uniquely determined $\mathbb{C}^{s \times s}$-valued measure.

Remark 3.4. If $r=(r(n))_{n \in \mathbb{Z}}$ is a $\mathbb{H}^{s \times s}$-valued sequence on $\mathbb{Z}$ such that

$$
r(n)=\int_{0}^{2 \pi} e^{i n t} d \nu(t)
$$

where $d \nu$ is a $q$-positive measure, then $r$ is Hermitian, i.e., $r(-n)^{*}=r(n)$.

The following result has been proved in [5, Theorem 5.5].

Theorem 3.5 (Herglotz's theorem for the quaternions). The function $n \mapsto r(n)$ from $\mathbb{Z}$ into $\mathbb{H}^{s \times s}$ is positive definite if and only if there exists a unique q-positive measure $\nu$ on $[0,2 \pi)$ such that

$$
r(n)=\int_{0}^{2 \pi} e^{i n t} d \nu(t), \quad n \in \mathbb{Z}
$$

Remark 3.6. For every $I \in \mathbb{S}$, there exists $J \in \mathbb{S}$ so that $I J=-J I$. Thus, $\mathbb{H}=$ $\mathbb{C}_{I} \oplus \mathbb{C}_{I} J$ and we may rewrite (3.4) as

$$
r(n)=\int_{0}^{2 \pi} e^{I n t} d \nu(t), \quad n \in \mathbb{Z}
$$

where $\nu=\nu_{1}+\nu_{2} J$ is a $q$-positive measure (in the sense that

$$
\mu=\left(\begin{array}{cc}
\nu_{1} & \nu_{2} \\
\nu_{2}^{*} & \nu_{3}
\end{array}\right)
$$

is positive). Here $\nu_{3}(t)=\nu_{1}(2 \pi-t)$.

For our purpose the scalar case will be important.

Lemma 3.7. If $U$ is a unitary operator on $\mathcal{H}$, then $r_{x}=\left(r_{x}(n)\right)_{n \in \mathbb{Z}}$, where $r_{x}(n)=$ $\left\langle U^{n} x, x\right\rangle$ for $x \in \mathcal{H}$, is an $\mathbb{H}$-valued positive definite sequence.

Proof. If $\left\{p_{0}, \ldots, p_{N}\right\} \subset \mathbb{H}$, then

$$
\begin{aligned}
\sum_{m, n=0}^{N} \bar{p}_{m} r_{x}(n-m) p_{n} & =\sum_{m, n=0}^{N} \bar{p}_{m}\left\langle U^{n-m} x, x\right\rangle p_{n} \\
& =\sum_{m, n=0}^{N}\left\langle U^{n-m} x p_{n}, x p_{m}\right\rangle \\
& =\sum_{m, n=0}^{N}\left\langle U^{n} x p_{n}, U^{m} x p_{m}\right\rangle \\
& =\left\langle\sum_{n=0}^{N} U^{n} x p_{n}, \sum_{m=0}^{N} U^{m} x p_{m}\right\rangle \\
& =\left\|\sum_{n=0}^{N} U^{n} x p_{n}\right\|^{2} \geq 0 .
\end{aligned}
$$

Thus, $r_{x}$ is a positive definite $\mathbb{H}$-valued sequence. 
Let $r_{x}$ be as in Lemma 3.7. It follows from Theorem 3.5 that there exists a unique $q$-positive measure $d \nu_{x}$ such that

$$
r_{x}(n)=\left\langle U^{n} x, x\right\rangle=\int_{0}^{2 \pi} e^{i n t} d \nu_{x}(t), \quad n \in \mathbb{Z} .
$$

One can check that

$$
\begin{aligned}
4\left\langle U^{n} x, y\right\rangle= & \left\langle U^{n}(x+y), x+y\right\rangle-\left\langle U^{n}(x-y), x-y\right\rangle+i\left\langle U^{n}(x+y i), x+y i\right\rangle \\
& -i\left\langle U^{n}(x-y i), x-y i\right\rangle+i\left\langle U^{n}(x-y j), x-y j\right\rangle k-i\left\langle U^{n}(x+y j), x+y j\right\rangle k \\
& +\left\langle U^{n}(x+y k), x+y k\right\rangle k-\left\langle U^{n}(x-y k), x-y k\right\rangle k
\end{aligned}
$$

and hence if we let

$$
\begin{aligned}
4 \nu_{x, y}:= & \nu_{x+y}-\nu_{x-y}+i \nu_{x+y i}-i \nu_{x-y i}+i \nu_{x-y j} k-i \nu_{x+y j} k \\
& +\nu_{x+y k} k-\nu_{x-y k} k
\end{aligned}
$$

then

$$
\left\langle U^{n} x, y\right\rangle=\int_{0}^{2 \pi} e^{i n t} d \nu_{x, y}(t), \quad x, y \in \mathcal{H} \quad \text { and } \quad n \in \mathbb{Z} .
$$

Theorem 3.8. The $\mathbb{H}$-valued measures $\nu_{x, y}$ defined on $\mathbf{B}([0,2 \pi))$ enjoy the following properties:

(i) $\nu_{x \alpha+y \beta, z}=\nu_{x, z} \alpha+\nu_{y, z} \beta, \quad \alpha, \beta \in \mathbb{H}$

(ii) $\nu_{x, y \alpha+z \beta}=\bar{\alpha} \nu_{x, y}+\bar{\beta} \nu_{x, z}, \quad \alpha, \beta \in \mathbb{C}_{i}$;

(iii) $\nu_{x, y}([0,2 \pi)) \leq\|x\|\|y\|$,

where $x, y, z \in \mathcal{H}$ and $\alpha, \beta \in \mathbb{H}$.

Proof. It follows from (3.9) that

$$
\begin{aligned}
\int_{0}^{2 \pi} e^{i n t} d \nu_{x \alpha+y \beta, z}(t) & =\left\langle U^{n} x, z\right\rangle \alpha+\left\langle U^{n} y, z\right\rangle \beta \\
& =\int_{0}^{2 \pi} e^{i n t}\left(d \nu_{x, z}(t) \alpha+d \nu_{y, z}(t) \beta\right), \quad n \in \mathbb{Z} .
\end{aligned}
$$

Use the uniqueness in Theorem 3.5 to conclude that

$$
\nu_{x \alpha+y \beta, z}(t)=\nu_{x, z}(t) \alpha+\nu_{y, z}(t) \beta
$$

and hence we have proved (i). Property (ii) is proved in a similar fashion, observing that $\bar{\alpha}, \bar{\beta}$ commute with $e^{\text {int }}$.

If $n=0$ in (3.9), then

$$
\langle x, y\rangle=\int_{0}^{2 \pi} d \nu_{x, y}(t)=\nu_{x, y}([0,2 \pi))
$$

and thus we can use an analog of the Cauchy-Schwarz inequality (see Lemma 5.6 in [11]) to obtain

$$
\nu_{x, y}([0,2 \pi)) \leq\|x\|\|y\|
$$

and hence we have proved (iii).

Remark 3.9. Contrary to the classical complex Hilbert space setting, $\nu_{x, y}$ need not equal $\bar{\nu}_{y, x}$. 
It follows from statements (i), (ii) and (iii) in Theorem 3.8 that $\phi(x)=\nu_{x, y}(\sigma)$, where $y \in \mathcal{H}$ and $\sigma \in \mathbf{B}([0,2 \pi))$ are fixed, is a continuous right linear functional. It follows from an analog of the Riesz representation theorem (see Theorem 6.1 in [11] or Theorem 7.6 in [13]) that corresponding to any $x \in \mathcal{H}$, there exists a uniquely determined vector $w \in \mathcal{H}$ such that

$$
\phi(x)=\langle x, w\rangle,
$$

i.e. $\nu_{x, y}(\sigma)=\langle x, w\rangle$. Use (i) and (ii) in Theorem 3.8 to deduce that $w=E(\sigma)^{*} y$. The uniqueness of $E$ follows readily from the construction. Thus, we have

$$
\nu_{x, y}(\sigma)=\langle E(\sigma) x, y\rangle, \quad x, y \in \mathcal{H} \quad \text { and } \quad \sigma \in \mathbf{B}([0,2 \pi)),
$$

whence

$$
\left\langle U^{n} x, y\right\rangle=\int_{0}^{2 \pi} e^{i n t}\langle d E(t) x, y\rangle
$$

To prove the main properties of the operator $E$ we need a uniqueness results on quaternionic measures which is a corollary of the following:

Theorem 3.10. Let $\mu$ and $\nu$ be $\mathbb{C}$-valued measures on $[0,2 \pi)$. If

$$
r(n)=\int_{0}^{2 \pi} e^{i n t} d \mu(t)=\int_{0}^{2 \pi} e^{i n t} d \nu(t), \quad n \in \mathbb{Z},
$$

then $\mu=\nu$.

Proof. See, e.g., Theorem 1.9.5 in [29].

Theorem 3.11. Let $\mu$ and $\nu$ be $\mathbb{H}$-valued measures on $[0,2 \pi)$. If

$$
r(n)=\int_{0}^{2 \pi} e^{i n t} d \mu(t)=\int_{0}^{2 \pi} e^{i n t} d \nu(t), \quad n \in \mathbb{Z},
$$

then $\mu=\nu$.

Proof. Write $r(n)=r_{1}(n)+r_{2}(n) j, \mu=\mu_{1}+\mu_{2} j$ and $\nu=\nu_{1}+\nu_{2} j$, where $r_{1}(n), r_{2}(n) \in$ $\mathbb{C}$ and $\mu_{1}, \mu_{2}, \nu_{1}, \nu_{2}$ are $\mathbb{C}$-valued measures on $[0,2 \pi)$. It follows from (3.13) that

$$
r_{1}(n)=\int_{0}^{2 \pi} e^{i n t} d \mu_{1}(t)=\int_{0}^{2 \pi} e^{i n t} d \nu_{1}(t), \quad n \in \mathbb{Z}
$$

and

$$
r_{2}(n)=\int_{0}^{2 \pi} e^{i n t} d \mu_{2}(t)=\int_{0}^{2 \pi} e^{i n t} d \nu_{2}(t), \quad n \in \mathbb{Z} .
$$

Use Theorem 3.10 to conclude that $\mu_{1}=\nu_{1}, \mu_{2}=\nu_{2}$ and hence that $\mu=\nu$.

Theorem 3.12. The operator E given in (3.10) enjoys the following properties:

(i) $\|E(\sigma)\| \leq 1$;

(ii) $E(\emptyset)=0$ and $E([0,2 \pi))=I_{\mathcal{H}}$;

(iii) If $\sigma \cap \tau=\emptyset$, then $E(\sigma \cup \tau)=E(\sigma)+E(\tau)$;

(iv) $E(\sigma \cap \tau)=E(\sigma) E(\tau)$;

(v) $E(\sigma)^{2}=E(\sigma)$;

(vi) $E(\sigma)$ commutes with $U$ for all $\sigma \in \mathbf{B}([0,2 \pi))$. 
Proof. Use (3.10) with $y=E(\sigma) x$ and (iii) in Theorem (3.8) to obtain

$$
\|E(\sigma) x\|^{2} \leq\|x\|\|E(\sigma) x\|
$$

whence we have shown (i). The first part of property (ii) follows directly from the fact that $\nu_{x, y}(\emptyset)=0$. The last part follows from (3.11) when $n=0$. Statement (iii) follows easily from the additivity of the measure $\nu_{x, y}$.

We will now prove property (iv). It follows from (3.11) that

$$
\begin{aligned}
\left\langle U^{n+m} x, y\right\rangle & =\int_{0}^{2 \pi} e^{i n t} e^{i m t}\langle d E(t) x, y\rangle \\
& =\left\langle U^{n}\left(U^{m} x\right), y\right\rangle \\
& =\int_{0}^{2 \pi} e^{i n t} d\left\langle E(t) U^{m} x, y\right\rangle .
\end{aligned}
$$

Using the uniqueness in Theorem 3.11 we obtain

$$
e^{i m t} d\langle E(t) x, y\rangle=\left\langle d E(t) U^{m} x, y\right\rangle
$$

and hence, denoting by $\mathbf{1}_{\sigma}$ the characteristic function of the set $\sigma$, we have

$$
\int_{0}^{2 \pi} \mathbf{1}_{\sigma}(t) e^{i m t}\langle d E(t) x, y\rangle=\left\langle E(\sigma) U^{m} x, y\right\rangle
$$

But

$$
\int_{0}^{2 \pi} \mathbf{1}_{\sigma}(t) e^{i m t}\langle d E(t) x, y\rangle=\left\langle U^{k} x, E(\sigma)^{*} y\right\rangle=\int_{0}^{2 \pi} e^{i m t} d\left\langle E(t) x, E(\sigma)^{*} y\right\rangle .
$$

Using the uniqueness in Theorem 3.11 once more we get

$$
\mathbf{1}_{\sigma}(t) d\langle E(t) x, y\rangle=\left\langle d E(t) x, E(\sigma)^{*} y\right\rangle
$$

and hence

$$
\int_{0}^{2 \pi} \mathbf{1}_{\tau}(t) \mathbf{1}_{\sigma}(t)\langle d E(t) x, y\rangle=\left\langle E(t) x, E(\sigma)^{*} y\right\rangle
$$

and thus

$$
\langle E(\sigma \cap \tau) x, y\rangle=\langle E(\sigma) E(\tau) x, y\rangle
$$

Property (v) is obtained from (iv) by letting $\sigma=\tau$.

Finally, since $U$ is unitary one can check that

$$
\left\langle U\left(x \pm U^{*} y\right), x \pm U^{*} y\right\rangle=\langle U(U x \pm y), U x \pm y\rangle
$$

and hence from (3.9) and the uniqueness in Theorem 3.11 we obtain $\nu_{x \pm U^{*} y}=\nu_{U x \pm y}$. Similarly,

$$
\begin{aligned}
& \nu_{x \pm U^{*} y i}=\nu_{U x \pm y i} \\
& \nu_{x \pm U^{*} y j}=\nu_{U x \pm y j}
\end{aligned}
$$

and

$$
\nu_{x \pm U^{*} y k}=\nu_{U x \pm y k}
$$

It follows from (3.8) that

$$
\nu_{x, U^{*} y}=\nu_{U x, y}
$$

Now use (3.10) to obtain

$$
\left\langle E(\sigma) x, U^{*} y\right\rangle=\langle E(\sigma) U x, y\rangle
$$


THE SPECTRAL THEOREM FOR UNITARY OPERATORS BASED ON THE S-SPECTRUM

i.e.,

$$
\langle U E(\sigma) x, y\rangle=\langle E(\sigma) U x, y\rangle, \quad x, y \in \mathcal{H}
$$

Given any quaternionic Hilbert space $\mathcal{H}$, there exists a subspace $\mathcal{M} \subset \mathcal{H}$ on $\mathbb{C}$ so that for any $x \in \mathcal{H}$ we have

$$
x=x_{1}+x_{2} j, \quad x_{1}, x_{2} \in \mathcal{M} .
$$

Theorem 3.13. Let $U$ be a unitary operator on a quaternionic Hilbert space $\mathcal{H}$ and let $E$ be the corresponding operator given by (3.10). E is self-adjoint if and only if $U: \mathcal{M} \rightarrow \mathcal{M}$, where $\mathcal{M}$ is as above.

Proof. If $E=E^{*}$, then it follows from (3.10) that $\nu_{x, y}=\bar{\nu}_{y, x}$ for all $x, y \in \mathcal{H}$. In particular, we get $\nu_{x, x}=\bar{\nu}_{x, x}$, i.e.

$$
\nu_{x}=\bar{\nu}_{x}, \quad x \in \mathcal{H} .
$$

Since $\nu_{x}$ is a $q$-positive measure we may write $\nu_{x}=\alpha_{x}+\beta_{x} j$, where $\alpha_{x}$ is a positive Borel measure on $[0,2 \pi)$ and $\beta_{x}$ is a complex Borel measure on $[0,2 \pi)$. It follows from (3.14) that

$$
\beta_{x}=-\beta_{x},
$$

i.e. $\beta_{x}=0$. Thus, we may make use of the spectral theorem for unitary operators on a complex Hilbert space (see, e.g., Section 31.7 in [27]) to deduce that $U: \mathcal{M} \rightarrow \mathcal{M}$. Conversely, if $U: \mathcal{M} \rightarrow \mathcal{M}$, then the spectral theorem for unitary operators on a complex Hilbert space yields that $E=E^{*}$.

If $U: \mathbb{H}^{n} \rightarrow \mathbb{H}^{n}$ is unitary, then (3.11) and Theorem 3.12 assert that

$$
U=\sum_{a=1}^{n} e^{i \theta_{a}} P_{a},
$$

where $\theta_{1}, \ldots, \theta_{n} \in[0,2 \pi)$ and $P_{1}, \ldots, P_{n}$ are oblique projections (i.e. $\left(P_{a}\right)^{2}=P_{a}$ but $\left(P_{a}\right)^{*}$ need not equal $\left.P_{a}\right)$. Corollary 6.2 in Zhang [33] asserts, in particular, the existence of $V: \mathbb{H}^{n} \rightarrow \mathbb{H}^{n}$ which is unitary and $\theta_{1}, \ldots, \theta_{n} \in[0,2 \pi)$ so that

$$
U=V^{*} \operatorname{diag}\left(e^{i \theta_{1}}, \ldots, e^{i \theta_{n}}\right) V .
$$

In the following remark we will explain how (3.15) and (3.16) are consistent.

Remark 3.14. Let $U: \mathbb{H}^{n} \rightarrow \mathbb{H}^{n}$ be unitary. Let $V$ and $\theta_{1}, \ldots, \theta_{n}$ be as above. If we let $e_{a}=(0, \ldots, 0,1,0, \ldots, 0)^{T} \in \mathbb{H}^{n}$, where the 1 is the $a$-th position, then we can rewrite 3.16 ) as

$$
U=\sum_{a=1}^{n} V^{*} e^{i \theta_{a}} e_{a} e_{a}^{*} V .
$$

Note that $V^{*} e^{i \theta_{a}} e_{a} e_{a}^{*} V=e^{i \theta_{a}} V^{*} e_{a} e_{a}^{*} V$ if and only if $V: \mathbb{C}^{n} \rightarrow \mathbb{C}^{n}$. In this case $U: \mathbb{C}^{n} \rightarrow \mathbb{C}^{n}$ and

$$
U=\sum_{a=1}^{n} e^{i \theta_{a}} P_{a},
$$

where $P_{a}$ denotes the orthogonal projection given by $V^{*} e^{i \theta_{a}} e_{a} e_{a}^{*} V$. 
Remark 3.15. Observe that in the proof of the spectral theorem for $U^{n}$ we have taken the imaginary units $i, j, k$ for the quaternions and we have determined spectral measures $\langle d E(t) x, y\rangle$ that are supported on the unit circle in $\mathbb{C}_{i}$. In the case one uses other orthogonal units $I, J$ and $K \in \mathbb{S}$ to represent quaternions, then the spectral measures are supported on the unit circle in $\mathbb{C}_{I}$.

Observe that (3.11) provides a vehicle to define a functional calculus for unitary operators on a quaternionic Hilbert space. For a continuous $\mathbb{H}$-valued function $f$ on the unit circle, which will be approximated by the polynomials $\sum_{k} e^{i k t} a_{k}$. We will consider a subclass of continuous quaternionic-valued functions defined as follows, see [23]:

Definition 3.16. The quaternionic linear space of slice continuous functions on an axially symmetric subset $\Omega$ of $\mathbb{H}$, denoted by $\mathcal{S}(\Omega)$ consists of functions of the form $f(u+I v)=\alpha(u, v)+I \beta(u, v)$ where $\alpha, \beta$ are quaternionic valued functions such that $\alpha(x, y)=\alpha(u,-v), \beta(u, v)=-\beta(u,-v)$ and $\alpha, \beta$ are continuous functions. When $\alpha, \beta$ are real valued we say that the continuous slice function is intrinsic. The subspace of intrinsic continuous slice functions is denoted by $\mathcal{S}_{\mathbb{R}}(\Omega)$.

It is important to note that any polynomial of the form $P(u+I v)=\sum_{k=0}^{n}(u+I v)^{n} a_{n}$, $a_{n} \in \mathbb{H}$ is a slice continuous function in the whole $\mathbb{H}$. A trigonometric polynomial of the form $P\left(e^{I t}\right)=\sum_{m=-n}^{n} e^{I m t} a_{m}$ is a slice continuous function on $\partial \mathbb{B}$, where $\mathbb{B}$ denotes the unit ball of quaternions.

Let us now denote by $\mathcal{P} \mathcal{S}\left(\sigma_{S}(T)\right)$ the set of slice continuous functions $f(u+I v)=$ $\alpha(u, v)+I \beta(u, v)$ where $\alpha, \beta$ are polynomials in the variables $u, v$.

In the sequel we will work on the complex plane $\mathbb{C}_{I}$ and we denote by $\mathbb{T}_{I}$ the boundary of $\mathbb{B} \cap \mathbb{C}_{I}$. Any other choice of an imaginary unit in the unit sphere $\mathbb{S}$ will provide an analogous result.

Remark 3.17. For every $I \in \mathbb{S}$, there exists $J \in \mathbb{S}$ so that $I J=-J I$. Bearing in mind Remark 3.6, we can construct $\nu_{x, y}^{(J)}$ so that (3.9) can also be written as

$$
\left\langle U^{n} x, y\right\rangle=\int_{0}^{2 \pi} e^{I n t} d \nu_{x, y}^{(J)}(t), \quad x, y \in \mathcal{H} \quad \text { and } \quad n \in \mathbb{Z} .
$$

Consequently, 3.11 can be written as

$$
\left\langle U^{n} x, y\right\rangle=\int_{0}^{2 \pi} e^{i n t}\left\langle E_{J}(t) x, y\right\rangle
$$

where $E_{J}$ is given by

$$
\nu_{x, y}^{(J)}(\sigma)=\left\langle E_{J}(\sigma) x, y\right\rangle, \quad x, y \in \mathcal{H} \quad \text { and } \quad \sigma \in \mathrm{B}\left(\mathbb{T}_{I}\right) .
$$

Moreover, $E_{J}$ satisfy properties (i)-(v) listed in Theorem 3.12 .

Theorem 3.18 (The spectral theorem for quaternionic unitary operators). Let $U$ be an unitary operator on a right linear quaternionic Hilbert space $\mathcal{H}$. Let $I, J \in \mathbb{S}, I$ orthogonal to $J$. Then there exists a unique spectral measure $E_{J}$ defined on the Borel sets of $\mathbb{T}_{I}$ such that for every slice continuous function $f \in \mathcal{S}\left(\sigma_{S}(U)\right)$, we have

$$
f(U)=\int_{0}^{2 \pi} f\left(e^{I t}\right) d E_{J}(t)
$$


Proof. Let us consider a polynomial $P(t)=\sum_{m=-n}^{n} e^{I m t} a_{m}$ defined on $\mathbb{T}_{I}$. Then using (3.18) we have

$$
\left\langle U^{m} x, y\right\rangle=\int_{0}^{2 \pi} e^{I m t}\left\langle d E_{J}(t) x, y\right\rangle \quad x, y, \in \mathcal{H} .
$$

By linearity, we can define

$$
\langle P(U) x, y\rangle=\int_{0}^{2 \pi} P\left(e^{I t}\right)\left\langle d E_{J}(t) x, y\right\rangle, \quad x, y, \in \mathcal{H} .
$$

The map $\Psi: \mathcal{P} \mathcal{S}\left(\sigma_{S}(U)\right) \rightarrow \mathcal{H}$ defined by $\psi_{U}(P)=P(U)$ is $\mathbb{R}$-linear. By fixing a basis for $\mathbb{H}$, e.g. the basis $1, i, j, k$, each slice continuous function $f$ can be decomposed using intrinsic function, i.e. $f=f_{0}+f_{1} i+f_{2} j+f_{3} k$ with $f_{\ell} \in \mathcal{S}_{\mathbb{R}}\left(\sigma_{S}(U)\right), \ell=0, \ldots, 3$, see [23, Lemma 6.11]. For these functions the spectral mapping theorem holds, thus $f_{\ell}\left(\sigma_{S}(U)\right)=\sigma_{S}\left(f_{\ell}(U)\right)$ and so $\left\|f_{\ell}(U)\right\|=\left\|f_{\ell}\right\|_{\infty}$, see [23, Theorem 7.4]. The map $\psi$ is continuous and so there exists $C>0$, that does not depend on $\ell$, such that

$$
\|P(U)\|_{\mathcal{H}} \leq C \max _{t \in \sigma_{S}(U)}|P(t)| \text {. }
$$

A slice continuous function $f \in \mathcal{S}\left(\sigma_{S}(U)\right)$ is defined on an axially symmetric subset $K \subseteq \mathbb{T}$ and thus it can be written as a function $f\left(e^{I t}\right)=\alpha(\cos t, \sin t)+I \beta(\cos t, \sin t)$ By fixing a basis of $\mathbb{H}$, e.g. $1, i, j, k, f$ can be decomposed into four slice continuous intrinsic functions $f_{\ell}(\cos t, \sin t)=\alpha_{\ell}(\cos t, \sin t)+I \beta_{\ell}(\cos t, \sin t), \ell=0, \ldots, 3$, such that $f=f_{0}+f_{1} i+f_{2} j+f_{3} k$.

By the Weierstrass approximation theorem for trigonometric polynomials, see, e.g., Theorem 8.15 in [28], each function $f_{\ell}$ can be approximated by a sequence of polynomials

$$
\tilde{R}_{\ell n}=\tilde{a}_{\ell n}(\cos t, \sin t)+I \tilde{b}_{\ell n}(\cos t, \sin t),
$$

$\ell=0, \ldots, 3$ which tend uniformly to $f_{\ell}$. These polynomials do not necessarily belong to the class of the continuous slice functions since $\tilde{a}_{\ell n}, \tilde{b}_{\ell n}$ do not satisfy, in general, the even and odd conditions in Definition 3.16. However, by setting

$$
\begin{gathered}
a_{\ell n}(u, v)=\frac{1}{2}\left(\tilde{a}_{\ell n}(u, v)+\tilde{a}_{\ell n}(u,-v)\right), \\
b_{\ell n}(u, v)=\frac{1}{2}\left(\tilde{b}_{\ell n}(u,-v)-\tilde{b}_{\ell n}(u, v)\right)
\end{gathered}
$$

we obtain that the sequence of polynomials $a_{\ell n}+I b_{\ell n}$ still converges to $f_{\ell}, \ell=0, \ldots, 3$. By putting $R_{\ell n}=a_{\ell n}(\cos t, \sin t)+I b_{\ell n}(\cos t, \sin t), \ell=0, \ldots, 3$ and $R_{n}=R_{0 n}+R_{1 n} i+$ $R_{2 n} j+R_{3 n} k$ we have a sequence of slice continuous polynomials $R_{n}\left(e^{I t}\right)$ converging to $f\left(e^{I t}\right)$ uniformly on $\mathbb{R}$.

By the previous discussion, $\left\{R_{n}(U)\right\}$ is a Cauchy sequence in the space of bounded linear operators since

$$
\left\|R_{n}(U)-R_{m}(U)\right\| \leq C \max _{t \in \sigma_{S}(U)}\left|R_{n}(t)-R_{m}(t)\right|,
$$

so as $R_{n}(U)$ has a limit which we denote by $f(U)$.

Remark 3.19. Fix $I \in \mathbb{S}$. It is worth pointing out that $f(u+I v)=(u+I v)^{-1}$ is an intrinsic function on $\mathbb{C}_{I} \cap \partial \mathbb{B}$, where $\partial \mathbb{B}=\{q \in \mathbb{H}:|q|=1\}$, since

$$
f(u+I v)=\frac{u}{u^{2}+v^{2}}+\left(\frac{-v}{u^{2}+v^{2}}\right) J .
$$


Thus, using Theorem 3.18, we may write

$$
U^{-1}=\int_{0}^{2 \pi} e^{-I t} d E_{J}(t)
$$

and

$$
U=\int_{0}^{2 \pi} e^{I t} d E_{J}(t)
$$

\section{The $S$-SPECTRUM AND THE SPECTRAL THEOREM}

We now want to show that the spectral theorem is based on the $S$-spectrum. We will be in need of the Cauchy formula for slice hyperholomorphic functions, see [17] for more details.

Definition 4.1 (Cauchy kernels). We define the (left) Cauchy kernel, for $q \notin[s]$, by

$$
S_{L}^{-1}(s, q):=-\left(q^{2}-2 q \operatorname{Re}(s)+|s|^{2}\right)^{-1}(q-\bar{s}) .
$$

We define the right Cauchy kernel, for $q \notin[s]$, by

$$
S_{R}^{-1}(s, q):=-(q-\bar{s})\left(q^{2}-2 \operatorname{Re}(s) q+|s|^{2}\right)^{-1} .
$$

Theorem 4.2. Let $\Omega \subseteq \mathbb{H}$ be an axially symmetric s-domain such that $\partial\left(\Omega \cap \mathbb{C}_{I}\right)$ is union of a finite number of continuously differentiable Jordan curves, for every $I \in \mathbb{S}$. Let $f$ be a slice hyperholomorphic function on an open set containing $\bar{\Omega}$ and, for any $I \in \mathbb{S}$, set $d s_{I}=-I d s$. Then for every $q=u+I_{q} v \in \Omega$ we have:

$$
f(q)=\frac{1}{2 \pi} \int_{\partial\left(\Omega \cap \mathbb{C}_{I}\right)} S_{L}(s, q) d s_{I} f(s) .
$$

Moreover, the value of the integral depends neither on $\Omega$ nor on the imaginary unit $I \in \mathbb{S}$.

If $f$ is a right slice regular function on a set that contains $\bar{\Omega}$, then

$$
f(q)=\frac{1}{2 \pi} \int_{\partial\left(\Omega \cap \mathbb{C}_{I}\right)} f(s) d s_{I} S_{R}^{-1}(s, q) .
$$

Moreover, the value of the integral depends neither on $\Omega$ nor on the imaginary unit $I \in \mathbb{S}$.

We conclude the paper with the following result, based on the Cauchy formula, that shows the relation between the spectral measures and the $S$-spectrum.

Theorem 4.3. Fix $I, J \in \mathbb{S}$, with $I$ orthogonal to $J$. Let $U$ be an unitary operator on a right linear quaternionic Hilbert space $\mathcal{H}$ and let $E(t)=E_{J}(t)$ be its spectral measure. Assume that $\sigma_{S}^{0}(U) \cap \mathbb{C}_{I}$ is contained in the arc of the unit circle in $\mathbb{C}_{I}$ with endpoints $t_{0}$ and $t_{1}$. Then

$$
\mathcal{P}\left(\sigma_{S}^{0}(U)\right)=E\left(t_{1}\right)-E\left(t_{0}\right) .
$$

Proof. The spectral theorem implies that the operator $S_{R}^{-1}(s, U)$ can be written as

$$
S_{R}^{-1}(s, U)=\int_{0}^{2 \pi} S_{R}^{-1}\left(e^{I t}, s\right) d E(t) .
$$


The Riesz projector is given by

$$
\mathcal{P}\left(\sigma_{S}^{0}(U)\right)=\frac{1}{2 \pi} \int_{\partial\left(\Omega_{0} \cap \mathbb{C}_{I}\right)} d s_{I} S_{R}^{-1}(s, U)
$$

where $\Omega_{0}$ is an open set containing $\sigma_{S}^{0}(U)$ and such that $\partial\left(\Omega_{0} \cap \mathbb{C}_{I}\right)$ is a smooth closed curve in $\mathbb{C}_{I}$. Now we write

$$
\mathcal{P}\left(\sigma_{S}^{0}(U)\right)=\frac{1}{2 \pi} \int_{\partial\left(\Omega_{0} \cap \mathbb{C}_{I}\right)} d s_{I}\left(\int_{0}^{2 \pi} S_{R}^{-1}\left(e^{I t}, s\right) d E(t)\right)
$$

and using the Fubini theorem we get

$$
\mathcal{P}\left(\sigma_{S}^{0}(U)\right)=\int_{0}^{2 \pi}\left(\frac{1}{2 \pi} \int_{\partial\left(\Omega_{0} \cap \mathbb{C}_{I}\right)} d s_{I} S_{R}^{-1}\left(e^{I t}, s\right)\right) d E(t) .
$$

It follows from the Cauchy formula that

$$
\frac{1}{2 \pi} \int_{\partial\left(\Omega_{0} \cap \mathbb{C}_{I}\right)} d s_{I} S_{R}^{-1}\left(e^{I t}, s\right)=\mathbf{1}_{\left[t_{0}, t_{1}\right]}
$$

where $\mathbf{1}_{\left[t_{0}, t_{1}\right]}$ is the characteristic function of the set $\left[t_{0}, t_{1}\right]$, and so we get the statement, since

$$
\mathcal{P}\left(\sigma_{S}^{0}(U)\right)=\int_{0}^{2 \pi} \mathbf{1}_{\left[t_{0}, t_{1}\right]} d E(t)=E\left(t_{1}\right)-E\left(t_{2}\right) .
$$

\section{REFERENCES}

[1] S. Adler, Quaternionic Quantum Field Theory, Oxford University Press, 1995.

[2] D. Alpay, The Schur algorithm, reproducing kernel spaces and system theory, American Mathematical Society, Providence, RI, 2001. Translated from the 1998 French original by Stephen S. Wilson, Panoramas et Synthèses.

[3] D. Alpay, F. Colombo, and I. Sabadini. Perturbation of the generator of a quaternionic evolution operator, to appear in Analysis and Applications (2014/15).

[4] D. Alpay, F. Colombo, J. Gantner, and I. Sabadini, A new resolvent equation for the S-functional calculus, arxiv 1310.7626v1, to appear in Journal of Geometric Analysis.

[5] D. Alpay, F. Colombo, D. P. Kimsey, and I. Sabadini. An extension of Herglotz's theorem to the quaternions, Preprint 2014, available on arxiv.

[6] D. Alpay, F. Colombo, I. Sabadini, Schur functions and their realizations in the slice hyperholomorphic setting, Integral Equations Operator Theory, 72 (2012), 253-289.

[7] D. Alpay, F. Colombo, I. Sabadini, Pontryagin De Branges Rovnyak spaces of slice hyperholomorphic functions, J. Anal. Math., 121 (2013), 87-125.

[8] D. Alpay, F. Colombo, I. Sabadini, Krein-Langer factorization and related topics in the slice hyperholomorphic setting, arXiv:1204.5491 to appear in Journal Geometric Analysis.

[9] D. Alpay, F. Colombo, and I. Sabadini. Inner product spaces and Krein spaces in the quaternionic setting, ArXiv e-prints, March 2013, to appear in Recent advances in inverse scattering, Schur analysis and stochastic processes. A collection of papers dedicated to Lev Sakhnovich, Operator Theory Advances and Applications. Linear Operators and Linear Systems, 2014.

[10] D. Alpay, A. Dijksma, J. Rovnyak, and H. de Snoo. Schur functions, operator colligations, and reproducing kernel Pontryagin spaces, volume 96 of Operator theory: Advances and Applications. Birkhäuser Verlag, Basel, 1997.

[11] D. Alpay and M. Shapiro. Reproducing kernel quaternionic Pontryagin spaces, Integral Equations and Operator Theory, 50:431-476, 2004.

[12] G. Birkhoff, J. von Neumann, The logic of quantum mechanics, Ann. of Math., 37 (1936), 823-843. 
[13] F. Brackx, R. Delanghe, F. Sommen, Clifford analysis, Research Notes in Mathematics, 76, Pitman, Advanced Publishing Program, Boston, MA, 1982.

[14] F. Colombo and I. Sabadini, The quaternionic evolution operator, Adv. Math., 227 (2011), 17721805.

[15] F. Colombo and I. Sabadini, On the formulations of the quaternionic functional calculus, J. Geom. Phys., 60 (2010), 1490-1508.

[16] F. Colombo, I. Sabadini, On some properties of the quaternionic functional calculus, J. Geom. Anal., 19 (2009), 601-627.

[17] F. Colombo, I. Sabadini, and D. C. Struppa. Noncommutative functional calculus. Theory and applications of slice hyperholomorphic functions., volume 289 of Progress in Mathematics, Birkhäuser/Springer Basel AG, Basel, 2011.

[18] N. Dunford, J. Schwartz. Linear Operators, part I: General Theory, J. Wiley and Sons (1988).

[19] N. Dunford, J. Schwartz. Linear Operators, part II: Spectral theory, J. Wiley and Sons (1988).

[20] G. Emch, Mécanique quantique quaternionienne et relativité restreinte, I, Helv. Phys. Acta, 36 (1963), 739-769.

[21] D. R. Farenick, B. A. F. Pidkowich, The spectral theorem in quaternions, Linear Algebra Appl., 371, (2003), 75-102.

[22] D. Finkelstein, J. M. Jauch, S. Schiminovich, D. Speiser, Foundations of quaternion quantum mechanics, J. Mathematical Phys. 3 (1962), 207-220.

[23] R. Ghiloni, V. Moretti, A. Perotti, Continuous slice functional calculus in the quaternionic Hilbert spaces, Rev. Math. Phys., 25 (2013), 1350006, 83 pp.

[24] R. Ghiloni, V. Moretti, A. Perotti, Spectral properties of compact normal quaternionic operators, Preprint 2014, arXiv:1402.2935v1.

[25] R. Ghiloni, V. Recupero, Semigroups over real alternative *-algebras: generation theorems and spherical sectorial operators, to appear in Trans. Amer. Math. Soc..

[26] L. P. Horwitz, L. C. Biedenharn, Quaternion quantum mechanics: Second quantization and gauge fields, Annals of Physics, 157 (1984), 43217488.

[27] P. D. Lax. Functional Analysis, J. Wiley and Sons (2002).

[28] W. Rudin, Principles of Mathematical Analysis, McGraw-Hill Inc., 1964.

[29] Z. Sasvári, Positive definite and definitizable functions, Mathematical Topics, Vol. 2, Akademie Verlag, Berlin, 1994.

[30] L. Schwartz. Sous espaces hilbertiens d'espaces vectoriels topologiques et noyaux associés (noyaux reproduisants), J. Analyse Math., 13 (1964), 115-256.

[31] Sharma, C. S. and Coulson, T. J., Spectral theory for unitary operators on a quaternionic Hilbert space, J. Math. Phys., 28, (1987), 1941-1946.

[32] K. Viswanath, Normal operators on quaternionic Hilbert spaces, Trans. Amer. Math. Soc., 162 (1971), 337-350.

[33] F. Zhang. Quaternions and matrices of quaternions, Linear Algebra Appl., 251 (1997), 21-57.

(DA) Department of Mathematics, Ben-Gurion University of the Negev, Beer-Sheva 84105 ISRAEL

E-mail address: dany@math.bgu.ac.il

(FC) Politecnico di Milano, Dipartimento di Matematica, Via E. Bonardi, 9,20133 Milano, Italy

E-mail address: fabrizio.colombo@polimi.it

(DPK)Department of Mathematics, Ben-Gurion University of the Negev, Beer-Sheva 84105 ISRAEL

E-mail address: dpkimsey@gmail.com

(IS) Politecnico di Milano, Dipartimento di Matematica, Via E. Bonardi, 9,20133 Milano, Italy

E-mail address: irene.sabadini@polimi.it 